2010

\title{
Magnetically insulated baffled probe for real-time monitoring of equilibrium and fluctuating values of space potentials, electron and ion temperatures, and densities
}

\author{
V. I. Demidov \\ M. E. Koepke \\ Y. Raitses
}

Follow this and additional works at: https://researchrepository.wvu.edu/faculty_publications

\section{Digital Commons Citation}

Demidov, V. I.; Koepke, M. E.; and Raitses, Y., "Magnetically insulated baffled probe for real-time monitoring of equilibrium and fluctuating values of space potentials, electron and ion temperatures, and densities" (2010). Faculty Scholarship. 221.

https://researchrepository.wvu.edu/faculty_publications/221 


\title{
Magnetically insulated baffled probe for real-time monitoring of equilibrium and fluctuating values of space potentials, electron and ion temperatures, and densities ${ }^{a)}$
}

\author{
V. I. Demidov, ${ }^{1}$ M. E. Koepke, ${ }^{1}$ and Y. Raitses ${ }^{2}$ \\ ${ }^{1}$ Department of Physics, West Virginia University, Morgantown, West Virginia 26506, USA \\ ${ }^{2}$ Princeton Plasma Physics Laboratory, Princeton, New Jersey 08543, USA
}

(Presented 19 May 2010; received 14 May 2010; accepted 2 August 2010; published online 28 October 2010)

\begin{abstract}
By restricting the electron-collection area of a cold Langmuir probe compared to the ion-collection area, the probe floating potential can become equal to the space potential, and thus conveniently monitored, rather than to a value shifted from the space potential by an electron-temperature-dependent offset, i.e., the case with an equal-collection-area probe. This design goal is achieved by combining an ambient magnetic field in the plasma with baffles, or shields, on the probe, resulting in species-selective magnetic insulation of the probe collection area. This permits the elimination of electron current to the probe by further adjustment of magnetic insulation which results in an ion-temperature-dependent offset when the probe is electrically floating. Subtracting the floating potential of two magnetically insulated baffled probes, each with a different degree of magnetic insulation, enables the electron or ion temperature to be measured in real time. (C) 2010 American Institute of Physics. [doi:10.1063/1.3490022]
\end{abstract}

Measurements of space potentials $\left(V_{s}\right)$ as well as electron/ion temperatures $\left(T_{e} / T_{i}\right)$ and densities $\left(N_{e}\right)$, and especially their oscillations $\left(\widetilde{V}_{s}, \widetilde{T}_{e}, \widetilde{T}_{i}\right.$, and $\left.\widetilde{N}_{e}\right)$ provide documentation of and insight into basic plasma properties, such as charged particle transport in plasmas. The measurements are particularly vital for fusion related plasmas, but also of great interest for technologically important low-temperature magnetized processing plasmas. ${ }^{1}$ For local real-time measurements of the above parameters and their oscillations, various types of electric probes can be used. ${ }^{2}$

Equilibrium values of $V_{s}, T_{e}$ and $N_{e}$ can be derived from data obtained by a simple Langmuir probe. ${ }^{2}$ Fast sweeping the probe gives us their local temporally resolved evolution. ${ }^{3}$ Using a floating Langmuir probe results in much higher temporal resolution, ${ }^{2}$ but the sensitivity of its electric potential to all three parameters makes it difficult to extract any one of them. An emissive floating probe minimizes this sensitivity, but released electrons can distort plasma and a hot probe filament has limited life time. Application of probe arrays can, in principle, distinguish different types of oscillations (like in a triple probe ${ }^{4}$ ), but signal coupling between emitted probes is responsible for spurious results.

The above probes cannot be used for measurements of ion temperatures. For that purpose the Katsumata probe has been developed. ${ }^{5}$ Typically, the fast sweeping Katsumata probe can resolve $T_{i}, V_{s}$, and $T_{e}$ evolutions but not oscillations because of the same limited temporal resolution as the fast sweeping Langmuir probe. This probe, like the plug probe $^{6}$ and the tunnel probe ${ }^{7}$ (essentially an inverse plug probe), is categorized as ion-sensitive probes because $T_{i}$ is

\footnotetext{
a) Contributed paper, published as part of the Proceedings of the 18th Topical Conference on High-Temperature Plasma Diagnostics, Wildwood, New Jersey, May 2010.
}

measured by suppressing the collected electron current.

Demidov et al. ${ }^{2,8,9}$ appears to be the first to suggest using similar probes in a floating regime for measurements of $\widetilde{V}_{s}$, electric field $\widetilde{E}_{s}$, and $\widetilde{T}_{i}$ in a magnetized plasma, which dramatically increases temporal resolution with respect to fast sweeping probes, and this paper summarizes its potential and achieved outcomes. It was demonstrated that, for plasmas with Maxwellian charged particle distributions, if the probe electron saturation current $I_{\text {sat }}^{e}$ is approximately equal to the ion saturation currant $I_{\text {sat, }}^{i}$, then the probe floating potential $V_{p} \approx V_{s}$. If $I_{\text {sat }}^{i} \gg I_{\text {sat }}^{e}$, then $V_{p} \approx V_{s}+\mu_{i} T_{i}$. In the opposite case $V_{p} \approx V_{s}-\mu_{e} T_{e}$. Here, $\mu_{i}=\ln \left(I_{\mathrm{sat}}^{i} / I_{\mathrm{sat}}^{e}\right)$ and $\mu_{e}=\ln \left(I_{\mathrm{sat}}^{e} / I_{\mathrm{sat}}^{i}\right)$.

For practical measurements of $\widetilde{V}_{s}$ and $\widetilde{E}_{s}$ the plug probes $^{10}$ and the baffled probes ${ }^{11}$ have been used. A simple Katsumata probe ${ }^{12}$ and a ball-pen probe ${ }^{13}$ (which is a slight modification of the Katsumata probe) have been applied for the measurements of $\widetilde{V}_{s}$. Later a novel ion-sensitive probe, which is basically a combination of the Katsumata probe and ion energy analyzer, was used by Ochoukov et al. ${ }^{14}$ Measurements of $\widetilde{T}_{i}$ have not been reported yet, but it is demonstrated that appropriate regime when $I_{\text {sat }}^{i} \gg I_{\text {sat }}^{e}$ is possible. ${ }^{9}$

The principles of operation of all these probes are similar and based on the dependence of the voltage drop in the plasma-probe sheath on the direction of the local magnetic field. When the magnetic field is parallel to the probe surface, the electron-repelling sheath can be significantly reduced as the magnetic field also impedes the cross-field electron flow and therefore, a smaller sheath voltage is needed to maintain the zero current balance to the floating probe. As a result, the accuracy of direct measurement of the plasma potential is greatly increased by eliminating the contribution of electron temperature and its fluctuations to the floatingpotential measurement. A set of baffles (or shields) blocking 
the electron flow to the probe tips placed at varying angles with respect to the magnetic field direction can enable a deduction of the plasma potential and the electron/ion temperature from the measured floating potentials of the different tips. Due to this all the above probes could be referred as baffled probes as it was proposed by Chen. ${ }^{15}$ All those probes can be also used as ion-sensitive probe with application of additional voltage for measurements of $T_{i}$. For measurements of plasma parameters in floating regime those probes require regulation of ratio between electron and ion currents and generally speaking can hardly be referred as ion-sensitive probes. For example, for measurements of $V_{s}$ the probes should be equally sensitive to ions and electrons.

Taking into account principles of the probe operation and the importance of the magnetic field for separation of electron and ion fluxes to the probe, it is preferable to use more accurate generic name. Due to this we propose to refer all those probes as magnetically insulated baffled (MIB) probe. Having a new more-descriptive label for this class of probe avoids confusion in general and specifically avoids misidentification when a term, such as baffle, is interpreted to mean the specific design ${ }^{11}$ when its intended use is to mean the general class of magnetically insulated probe.

For the practical implementation and development of a specific design of the MIB probe, several issues have to be taken into account. It is clear that the probe modeling (which is important for obtaining more reliable information) depends strongly on specific shape of the probe. For example, it is known that the plug probe has more favorable geometry for modeling than the simple Katsumata probe. ${ }^{16}$ The MIB probe in the form of the ball-pen probe has even more complicated shape than the Katsumata probe and probably is more difficult for modeling. At the same time, the plug probe is more difficult to align and it is less reliable for harsh fusion plasma conditions. For those conditions the simplicity of the probe construction is an important issue which gives robustness of creation of simple arrays, which is crucial for electric fields and temperature measurements. Another issue is a possibility to make measurements of $T_{i}$ fluctuations. To do that the above condition $I_{\text {sat }}^{i} \gg I_{\text {sat }}^{e}$ has to be achieved. That probably depends on diffusion of charged particles near the probe. It looks like the diffusion should be classical to achieve that regime. A possibility to get that depends on probe size and construction. Note here that global diffusion in a plasma may be anomalous, but local near-probe diffusion may be classical at the same time. ${ }^{2}$

Below we provide as an illustration some results of measurements of $\widetilde{V}_{s}, \widetilde{T}_{e}$, and $\widetilde{E}_{s}$ in plasma waves in the steadystate barium plasma column (3 m length) of the West Virginia University Q-machine. ${ }^{11}$ The experimental setup is shown in Fig. 1. Plasma was produced by surface ionization on a grounded, hot, rhenium-coated tungsten plate (1). At the opposite end of the device, at the distance of $10 \mathrm{~cm}$ before the terminating electrode (4), a $2.5 \mathrm{~cm}$ diameter biasable mesh-electrode (3) was placed perpendicular to the cylindrical axis. The typical electron and ion temperatures are $T_{e}$ $\approx T_{i} \approx 0.2 \mathrm{eV}$; magnetic fields are $B \sim 0.5-3.0 \mathrm{kG}$. Average Larmor radius of electrons $R_{L e}$ for $B=3 \mathrm{kG}$ is $0.005 \mathrm{~mm}$ and for ions $R_{L i}$ is $2.8 \mathrm{~mm}$.

The oscillation measurements were conducted with the probe cluster (2), ${ }^{17}$ which was at the distance of $175 \mathrm{~cm}$ from

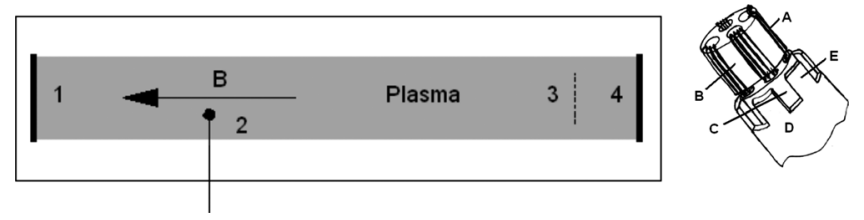

FIG. 1. Schematic diagram of WVU Q-machine (left): the grounded hot plate (1), the four-probe cluster (2), the mesh-electrode (3), and a solid terminating electrode (4). Sketch of the probe cluster (right): collection wire (a), short four-bore ceramic tube (b), four-bore ceramic tube (c), outer single-bore ceramic tube (d), and baffles (e). In the sketch, the four-bore tubes of the cluster shifted out of outer tube to demonstrate the probe construction.

the mesh. The probe cluster had the following dimensions. Outer radius of the four-bore tube (2) was $1.4 \mathrm{~mm}$ and radius of each bore was $0.25 \mathrm{~mm}$. The four-bore tube length was $10 \mathrm{~mm}$. The outer radius of the single bore tube was $3.2 \mathrm{~mm}$ and its inner radius was $1.6 \mathrm{~mm}$. The dimensions of each gap in the outer tube were $2 \mathrm{~mm}$ (width) and $5 \mathrm{~mm}$ (length). Gold wire with diameter of $0.1 \mathrm{~mm}$, being soft, was used for the collection wire in order to have coil corners that hug the ceramic tube corners. Each coil was created by four windings of gold wire. The cluster axis was oriented perpendicular to magnetic field. For the measurements reported here, the probe cluster was rotated so that two diametrically opposite probe tips intersected the same magnetic line. For this probecluster orientation, these two baffled probes are referred to as "open" since electrons had full access to the probe (from one direction). One open probe faces the plasma source (1) and the other open probe faces the mesh (3). The other two baffled probes in the cluster are referred to as "closed" since electron access to the probe was minimal. More details about the probe cluster can be found in Ref. 17.
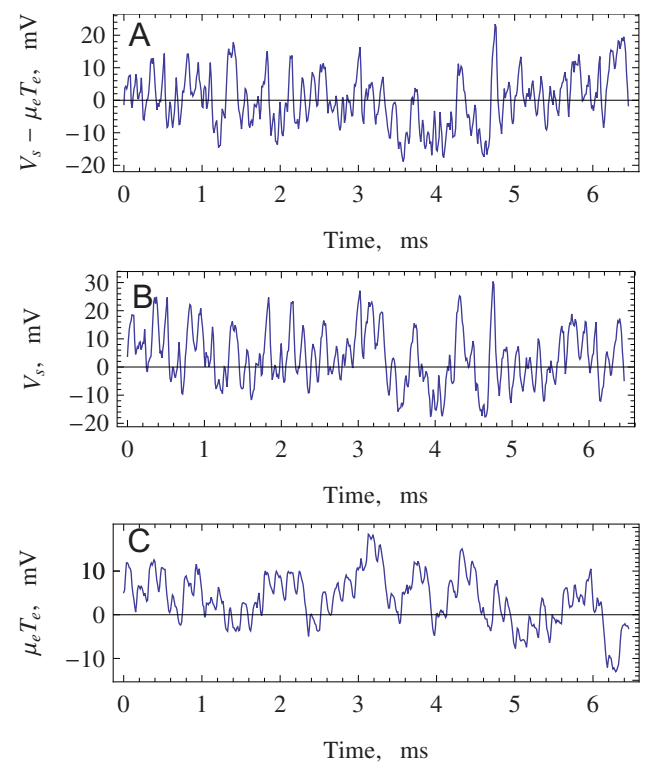

FIG. 2. (Color online) Time series of ac-coupled baffled-probe signals. (a) Half sum of two adjacent open-probe signals. This quantity is interpreted as $\widetilde{V}_{s}-\mu_{e} \widetilde{T}_{e}$. (b) Half sum of two adjacent closed-probe signals. This quantity is interpreted as $\widetilde{V}_{s}$. (c) Difference signal between the half sum of two adjacent closed-probe signals and the half sum of two adjacent open-probe signals. This quantity is interpreted as $\mu_{e} \widetilde{T}_{e}$. 


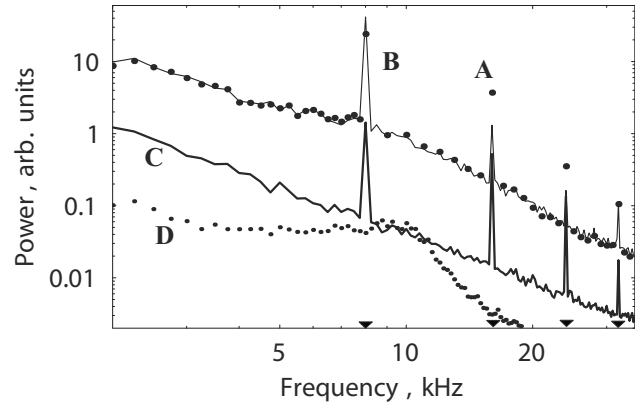

FIG. 3. Power spectra of $\widetilde{V}_{p}$ for open (big dots A) and closed (curve B) probes. Power spectrum of $\bar{\mu}_{e} \widetilde{T}_{e}$ oscillations (curve C). Power spectrum of $\widetilde{V}_{p}$ for open probe in the plasma without modulation of the mesh bias (small dots D). Frequency of $8 \mathrm{kHz}$ and its harmonics marked by triangles at $x$-axis.

To obtain the $\mu_{e} \widetilde{T}_{e}$ spectrum, a half of sum of two closed-probe signals was calculated, giving us oscillations of $\widetilde{V}_{s}$ at the centroid of the probe tips. The similar half of sum of signals of two open probes gives us oscillations of $\tilde{V}_{s}$ $-\bar{\mu}_{e} \widetilde{T}_{e}$ at the same point, where $\bar{\mu}_{e} \approx 5$ is the average of $\mu_{e}$ for both open probes. Then, subtracting the second signal from the first one we can obtain oscillations of $\bar{\mu}_{e} \widetilde{T}_{e}$, corresponding to a single point in the plasma. This procedure minimizes uncertainties that arise when we use signals from probes situated at different locations in the plasma. Of course this procedure is correct if the relative phase between signals is small, which is true in our case due to the small distance between probe tips relative to the wavelength (as checked by cross-phase measurements below).

Measurements have been repeated for the four similar orientations (rotations in $90^{\circ}$ increments) to check reproducibility of the results when each pair of diametrically opposite probe tips has been alternately open and closed. During the measurements, 50000 samples of floating probe potential from each probe of one cluster are sampled simultaneously at $100 \mathrm{kHz}$. Any channel of the four-channel measurement circuit could be connected to any probe. That allows us to verify possible pickups from an ac generator.

Examples of time-based signals from the probe cluster are shown in Fig. 2. As described above, the two open-probe signals are averaged to obtain Fig. 2(a), the time series of the fluctuating floating potential of an open probe located effectively midway between the two open-probe tips. Likewise,

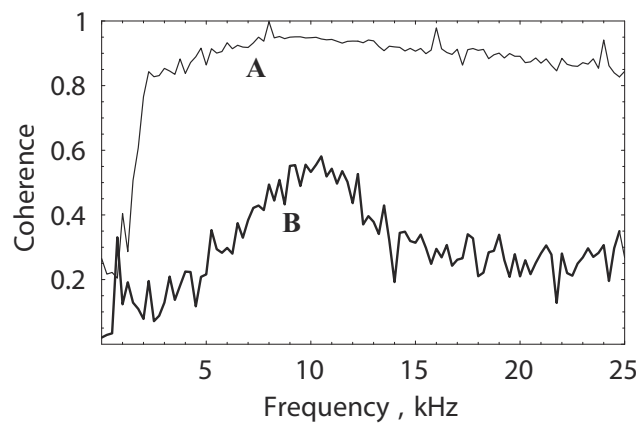

FIG. 4. Coherency between two closed-probe potential oscillations with application of ac voltage to the mesh (curve A) and without this bias modulation (curve B).

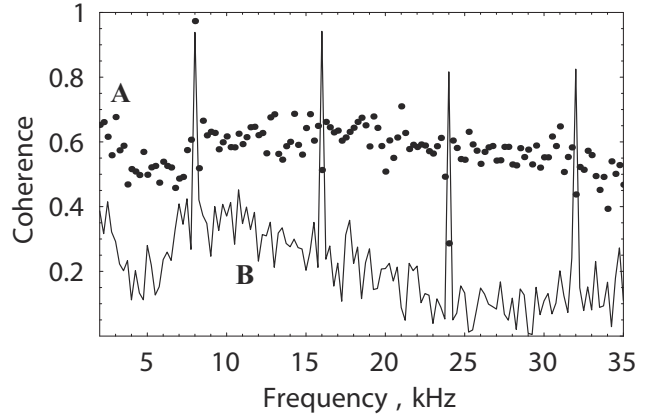

FIG. 5. Coherency between plasma potential oscillation and $\widetilde{E}_{y}$ (dots A); electron temperature and plasma potential oscillations (curve B).

the two closed-probe signals are averaged to obtain Fig. 2(b). Figure 2(b) is interpreted as the fluctuating, midpoint, space potential $\widetilde{V}_{s}$. In Figs. 2(a) and 2(b), the small differences in amplitude and phase, attributed to temperature fluctuations, are isolated in Fig. 2(c) by subtracting Fig. 2(a) from Fig. 2(b). Figure 2(c) is interpreted as $\mu_{e} \widetilde{T}_{e}$, where $\mu_{e} \approx 5$. Those examples are corresponding to the experiments shown in Figs. 3-8.

All presented measurements have been conducted at $1 \mathrm{~cm}$ from the cylindrical axis and $B=3 \mathrm{kG}$. Without the mesh (3) ac modulation, the turbulence was much less exaggerated, as evidenced in Fig. 3 (small dots D). Figure 4 shows coherency between two closed-probe potential oscillations essentially coherent between $\widetilde{V}_{s}$ in two separated points of the plasma. As we can also see [Fig. 4(b)], for this case, the coherency is low and that confirms the absence of developed turbulence and indicates that the oscillations are mostly noise.

After application of ac voltage of amplitude of $70 \mathrm{~V}$ and frequency of $8 \mathrm{kHz}$ to the mesh (3), the amplitude of oscillations sharply increases. Figure 3 demonstrates the power spectrum of floating potential of an open (faces to the source) probe (big dots A), which gives us $\widetilde{V}_{s}-\mu_{e} \widetilde{T}_{e}$. As we can also see from Fig. 4(a), in this case coherency is much higher at all frequencies for exception low frequencies $(<2 \mathrm{kHz})$. Therefore, in this case there is a developed turbulence.

In the same Fig. 3, the power spectrum of plasma potential oscillations measured by one of the closed probes (upper solid line B) is presented. Note that both spectra (measured

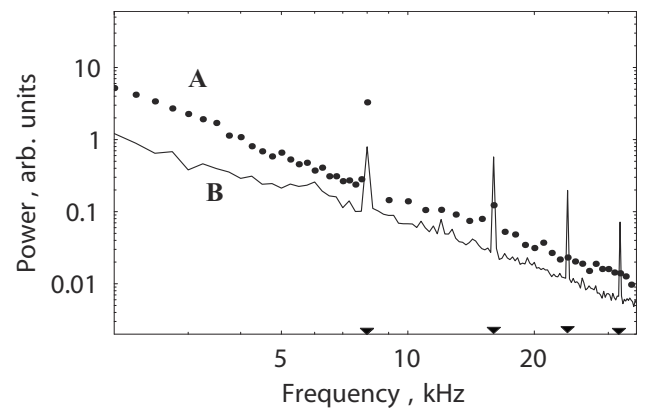

FIG. 6. Power spectrum of electric field component $\widetilde{E}_{y}$ perpendicular to the magnetic field in azimuthal direction (dots A). Power spectrum of electric field component $\widetilde{E}_{z}$ along the magnetic field (curve B). Frequency of $8 \mathrm{kHz}$ and its harmonics marked by triangles at $x$-axis. 


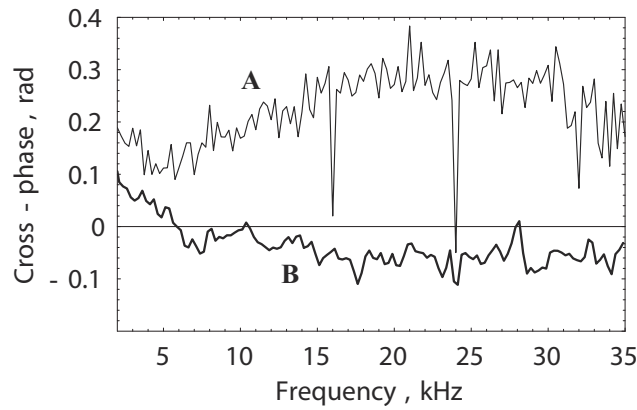

FIG. 7. Phase between space potential oscillations perpendicular to the magnetic field in azimuthal direction (closed probes, curve A). Phase between floating probe oscillations in the direction parallel to the magnetic field (open probes, curve B).

by open and closed probes) practically coincide with each other, except at the exciting frequency of $8 \mathrm{kHz}$ and its harmonics $(16,24$, and $32 \mathrm{kHz})$, which can be evidence of the insignificance of temperature oscillations at all frequencies except the harmonics of $8 \mathrm{kHz} .{ }^{9,17}$ Note that it was shown in Ref. 9 that spectra $\widetilde{V}_{s}-\mu_{e} \widetilde{T}_{e}$ and $\widetilde{V}_{s}$ in Fig. 3 could be obtained by a single baffled probe; however, the probe cluster allows us to extract power spectrum of $\mu_{e} \widetilde{T}_{e}$ from the measurements and to measure cross-phases between oscillations.

The obtained power spectra of $\bar{\mu}_{e} \widetilde{T}_{e}$ are also presented in Fig. 3 (lower solid curve C). The temperature oscillations are negligible in the broad spectrum. To prove that, Fig. 5 demonstrates coherency between plasma potential oscillations $\tilde{V}_{s}$ and electron temperature oscillations $\widetilde{T}_{e}$ (lower solid curve B). The coherency is low at frequencies associated with the broad spectrum. It means that the measured $\widetilde{T}_{e}$ do not associate with $\widetilde{V}_{s}$ and are mostly noise. On the other hand, temperature fluctuations are more important at frequencies of 8, 16,24 , and $32 \mathrm{kHz}$, where power of $\widetilde{V}_{s}$ oscillation is close to power of $\mu_{e} \widetilde{T}_{e}$ oscillations. For those frequencies coherency between $\widetilde{T}_{e}$ and $\widetilde{V}_{s}$ is high, so they belong to the same mode. Note also that power spectra of $\widetilde{V}_{s}, \widetilde{V}_{s}-\bar{\mu}_{e} \widetilde{T}_{e}$, and $\mu_{e} \widetilde{T}_{e}$ at frequencies of $8,16,24$, and $32 \mathrm{kHz}$ had very good reproducibility during rotation of the probe cluster by $90^{\circ}$ to check the influence of different probe tips and possible small misalignment as it was described above, but change in measured broad spectrum of $\mu_{e} \widetilde{T}_{e}$ could be up to a few times.

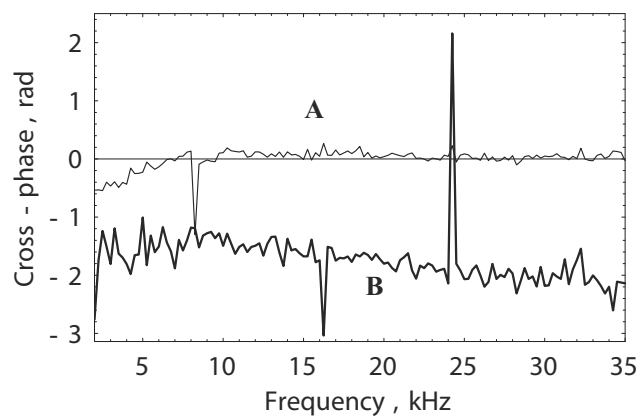

FIG. 8. Phase between space potential oscillations and $\widetilde{E}_{z}$ (curve A). Phase between space potential oscillations and $\widetilde{E}_{y}$ (curve B).
Absolute values of amplitude of $\bar{\mu}_{e} \widetilde{T}_{e}$ oscillation narrow peaks are $1,0.6,0.3$, and $0.1 \mathrm{mV}$ corresponding to frequencies of $8,16,24$, and $32 \mathrm{kHz}$. To obtain absolute values of amplitude of $\widetilde{T}_{e}$, we should divide the above values by factor of $\mu_{e} \approx 5$. Those correspond to $2.3,1.4,0.7$, and $0.2 \mathrm{~K}$, respectively. This accuracy, and for relative measurements only, can be obtained from floating probes which can easily measure signals of order of a fraction of $1 \mathrm{mV}$.

The difference signal from two closed probes allows us to obtain power spectrum of the component of electric field $\widetilde{E}_{y}$ perpendicular to the magnetic field in azimuthal direction. The result of taking this difference is presented in Fig. 6 (dots A). The component $\widetilde{E}_{y}$ has broad power-law spectrum and practically only one maximum at $8 \mathrm{kHz}$ (a harmonic at $16 \mathrm{kHz}$ is very small and harmonics at 24 and $32 \mathrm{kHz}$ are nondistinguishable). This maximum corresponds to amplitude of $\widetilde{E}_{y}$ of $0.2 \mathrm{~V} / \mathrm{m}$. Figure $7(\mathrm{a})$ shows cross-phase between two closed probes. The behavior of the cross-phase of azimuthally propagating oscillations is typical for drift waves. ${ }^{1}$ In contrast, at frequencies of 16,24 , and $32 \mathrm{kHz}$, the phase shifts are sharply decreased. This is because waves in axial direction are dominant (phase shift in azimuthal direction for axially propagating waves is zero).

Measurements of cross-phase between electric potential oscillations and components of electric field oscillations (see Fig. 8) reinforce our interpretation. Cross-phase between $\widetilde{E}_{y}$ and $\widetilde{V}_{s}$ is close to $-\pi / 2$ for almost all frequencies, with exception of harmonics 16 and $24 \mathrm{kHz}$, where $\widetilde{V}_{s}$ and $\widetilde{T}_{e}$ belong to the different mode.

As we can see from Fig. 5(a), coherency between $\widetilde{E}_{y}$ and $\widetilde{V}_{s}$ is sufficiently high (0.6) for broad spectrum, goes up for 8 $\mathrm{kHz}$, but drops down at harmonics (that is especially visible at $24 \mathrm{kHz}$ ). It confirms that fluctuations of space potential at frequency of $8 \mathrm{kHz}$ have contributions from the same mode as $\widetilde{E}_{y}$, but other harmonics belong to the different mode.

The difference signal from two open probes that lie on the same magnetic field line could give us the component of electric field $\widetilde{E}_{z}$ in the direction parallel to the magnetic field (axial direction). However, in this case measurement of cross-phase between two open probes gives us very small angles close to zero [see Fig. 8(a)]. It means that the calculated difference (Fig. 6, lower curve B) has big errors and can serve as an upper limit for $\widetilde{E}_{z}$. Nevertheless, it is clear that $\widetilde{E}_{y} \gg \widetilde{E}_{z}$, like it could be in drift waves. ${ }^{18,19}$ At the harmonics of $8 \mathrm{kHz}$, the signals being subtracted include temperature fluctuations that contaminate the $\widetilde{E}_{z}$ calculation. Due to this, the power spectrum $\widetilde{E}_{z}(\nu)$ has a number of well pronounced harmonics with power considerably higher than the power in $\widetilde{E}_{y}(\nu)$. In Fig. 8(a) an example of measurements of cross-phases between $\widetilde{V}_{s}$ and $\widetilde{E}_{z}$ is presented. To distinguish more precisely between axial components at temperature and potential oscillations, measurements with two closed probes spaced further apart (or with two axially spaced probe clusters) are required.

Thus, our investigation of power spectra of $\widetilde{V}_{s}, \widetilde{T}_{e}$, and $\widetilde{E}_{y, z}$ suggests the presence of two modes of oscillation. One mode has a broad spectrum of potential oscillations without 
significant temperature oscillations. This may correspond to drift-acoustic waves. The second mode has associated with it narrow-band peak oscillations of parallel electric field at harmonics of $8 \mathrm{kHz}$. It seems that this mode has associated with fluctuations in the ac current collected by the mesh (3) and Joule heating fluctuations arise from this current.

The authors are grateful to S. M. Finnegan and E. W. Reynolds for their help in experiments. This work was supported by the DOE OFES (Contract No. DE-SC0001939) and AFOSR.

${ }^{1}$ F. F. Chen, Introduction to Plasma Physics and Controlled Fusion, 2nd ed. (Plenum, New York, 1983).

${ }^{2}$ V. I. Demidov, S. V. Ratynskaia, and K. Rypdal, Rev. Sci. Instrum. 73, 3409 (2002).

${ }^{3}$ B. LaBombard and L. Lyons, Rev. Sci. Instrum. 78, 073501 (2007).

${ }^{4}$ S.-L. Chen and T. Sekiguchi, J. Appl. Phys. 36, 2363 (1965).

${ }^{5}$ I. Katsumata and M. Okazaki, Jpn. J. Appl. Phys., Part 1 6, 123 (1967).

${ }^{6}$ V. I. Demidov, S. V. Ratynskaia, and K. Rypdal, Rev. Sci. Instrum. 70, 4266 (1999).

${ }^{7}$ J. P. Gunn, P. Devynck, J.-Y. Pascal, J. Adámek, I. Ďuran, M. Hron, J. Stöckel, F. Žáček, O. Bařina, and R. Hrach, Czech. J. Phys. 52, 1107 (2002).
${ }^{8}$ V. I. Demidov, S. V. Ratynskaia, and K. Rypdal, International Topical Conference on Plasma Physics: New Frontiers of Nonlinear Science, Faro, Portugal, 1999

${ }^{9}$ V. I. Demidov, S. M. Finnegan, M. E. Koepke, and E. W. Reynolds, Contrib. Plasma Phys. 44, 689 (2004).

${ }^{10}$ S. V. Ratynskaia, V. I. Demidov, and K. Rypdal, Rev. Sci. Instrum. 71, 1367 (2000).

${ }^{11}$ V. I. Demidov, S. M. Finnegan, M. E. Koepke, and E. W. Reynolds, Rev. Sci. Instrum. 74, 4558 (2003).

${ }^{12}$ F. Greiner, D. Block, A. Piel, and S. Ratynskaia, "Plasma potential measurement in a magnetised plasma," http://old.dpg-tagungen.de/archive/ 2003/p_10.html, 2003.

${ }^{13}$ R. Schrittwieser, C. Ionită, P. C. Balan, A. F. Varandas, H. F. C. Figueiredo, J. Stöckel, J. Adámek, M. Hron, J. Ryszawy, M. Tichý, E. Martines, G. Van Oost, T. Klinger, and R. Madani, Rom. J. Physiol. 50, 723 (2005).

${ }^{14}$ R. Ochoukov, D. G. Whyte, B. Lipschultz, B. LaBombard, and S. Wukitch, Rev. Sci. Instrum. 81, 10E111 (2010).

${ }^{15}$ F. F. Chen, IEEE-ICOPS Meeting, Jeju, Korea, 2003.

${ }^{16}$ I. Hutchinson, Principles of Plasma Diagnostics (Cambridge University Press, Cambridge, England, 2005).

${ }^{17}$ M. E. Koepke, V. I. Demidov, S. M. Finnegan, and E. W. Reynolds, Contrib. Plasma Phys. 46, 385 (2006).

${ }^{18}$ F. F. Chen, Phys. Fluids 10, 1647 (1967).

${ }^{19}$ R. F. Ellis, E. Marden-Marshall, and R. Majeski, Plasma Phys. 22, 113 (1980). 